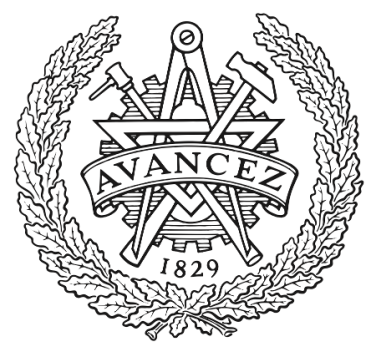

CHALMERS

UNIVERSITY OF TECHNOLOGY

\title{
An extended kalman filter framework for joint phase noise, CFO and sampling time error estimation
}

Downloaded from: https://research.chalmers.se, 2023-04-26 15:29 UTC

Citation for the original published paper (version of record):

Hajiabdolrahim, N., Rezaei Aghdam, S., Eriksson, T. (2020). An extended kalman filter framework for joint phase noise, $\mathrm{CFO}$ and sampling time error estimation. IEEE International Symposium on Personal, Indoor and Mobile Radio Communications, PIMRC, 2020-August. http://dx.doi.org/10.1109/PIMRC48278.2020.9217229

N.B. When citing this work, cite the original published paper. 


\section{An Extended Kalman Filter Framework for Joint Phase Noise, CFO and Sampling Time Error Estimation}

\author{
Nima Hajiabdolrahim \\ Department of Electrical Engineering \\ Chalmers University of Technology \\ Gothenburg, Sweden \\ nimahaj@chalmers.se
}

\author{
Sina Rezaei Aghdam \\ Department of Electrical Engineering \\ Chalmers University of Technology \\ Gothenburg, Sweden \\ sinar@chalmers.se
}

\author{
Thomas Eriksson \\ Department of Electrical Engineering \\ Chalmers University of Technology \\ Gothenburg, Sweden \\ thomase@chalmers.se
}

\begin{abstract}
We present a framework for joint estimation and compensation of three major oscillator impairments, namely sampling time error (STE), carrier frequency offset (CFO) and phase noise (PN). In particular, we model these impairments as Wiener processes and introduce a pilot-aided approach which facilitates their joint estimation. The proposed solution is carried out in two steps: first, an initial estimation of the transmitted symbols is acquired by applying an extended Kalman filter (EKF) on the pilot symbols and then, a second EKF is applied on the estimated symbols which yields an accurate tracking of STE, PN and CFO over an additive white Gaussian noise channel. Our numerical results demonstrate the efficacy of the proposed solution.
\end{abstract}

Index Terms-Carrier frequency offset, extended Kalman filter, phase noise, sampling time error, synchronization.

\section{INTRODUCTION}

The performance of any communication system is fundamentally limited by the impairments introduced by nonideal hardware. A successful demodulation of transmitted data relies on processes of tracking and compensation of different hardware impairments at the receiver side. Timing synchronization is among these crucial tasks.

Timing synchronization is referred to the process of determining the correct time instances at which the received signal should be sampled. The timing recovery may not be a difficult task by itself and several classic solutions have addressed it, see, e.g., [1] for an overview. What makes timing recovery challenging is that sample time offsets occur in conjunction with other hardware impairments such as carrier frequency offset (CFO).

Joint estimation of sample time offset and CFO has been addressed in few research articles. For instance, [2] proposes a joint blind estimation technique which relies on sequential importance sampling particle filter. The authors in [3] develop a data-aided maximum likelihood algorithm for joint CFO and sample time offset estimation. Their two-step approach

This research has been carried out in the GigaHertz centre in a joint research project financed by Swedish Governmental Agency of Innovation Systems (VINNOVA), Chalmers University of Technology, Ericsson, SAAB and RUAG. relies on estimating $\mathrm{CFO}$ via two FFT operations and then, computing sample time offset using a simple closed-form expression.

Phase noise $(\mathrm{PN})$ is the other important hardware impairment which can hinder the synchronization process and if not properly compensated, can considerably deteriorate the reception. Our objective in this paper is to address joint estimation and compensation of sample time offset, $\mathrm{CFO}$ and phase noise over an additive white Gaussian noise (AWGN) channel. One may note that these hardware impairments are highly coupled, i.e., they affect each other and get affected by each other. As a result a proper estimation of them should be performed jointly. Such joint estimation to the best of our knowledge has not been addressed before.

In this paper, we introduce a pilot-aided scheme for jointly tracking sample time offset, phase noise and frequency offset. We model sampling time error (STE) and PN using a Wiener random process and, we propose an extended Kalman filter-aided framework for their joint estimation. Our proposed solution is different from the conventional timing synchronization techniques [5] in many aspects. First of all, in the presented framework, STE is modeled using a more accurate model, namely, via Wiener process. Moreover, a clear advantage of the proposed estimation technique is that does not require oversampling.

Section II describes our system model of interest. In Section III, we explain our proposed solution. We demonstrate the efficacy of the proposed scheme through numerical examples in Section IV and, the paper is concluded in Section $\mathrm{V}$.

\section{SYSTEM MODEL}

The system model which will be considered in this paper is depicted in Figure 1. PN and CFO effects can be represented by a phase rotation in the received symbols, i.e., if the $n$-th transmitted symbol is shown by $x_{n}$, then taking into account just PN and CFO, the $n$-th received symbol can be expressed as $y_{n}=e^{j \Phi_{n}} x_{n}+w_{n}$ for $n=1,2, \cdots, N$, where $w_{n}$ denotes the AWGN, $\Phi_{n}$ is the total phase shift due to PN and CFO 


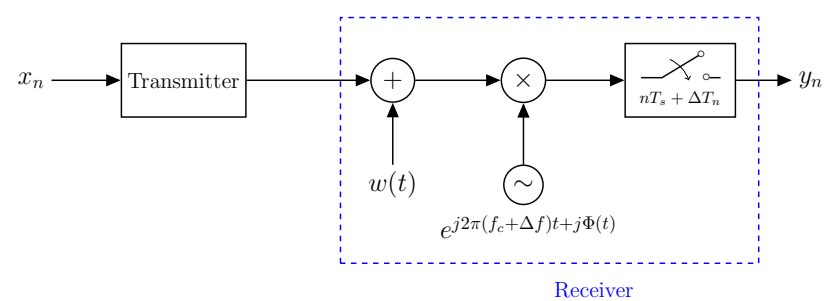

Fig. 1. System model of an AWGN communication link experiencing carrier frequency offset (represented by $\Delta f$ ), phase noise (represented by $\Phi(t)$ in continuous time domain) and sampling time error (represented by $\Delta T_{n}$ ) at the receiver side.

and $N$ is the number of transmitted symbols. Considering the characteristics of PN and CFO [4], one can model $\Phi_{n}$ using a Wiener process which can be expressed as follows

$$
\Phi_{n}=\Phi_{n-1}+\Delta f+\xi_{n}, \quad n=2,3, \cdots, N,
$$

where $\xi_{n} \sim \mathcal{N}\left(0, \sigma_{\xi}^{2}\right)$ are independent and identically distributed (i.i.d.) Gaussian random variables and $\Delta f$ denotes the frequency offset effect, which is usually assumed to be a constant value.

However, STE produces a more significant effect on the received symbols. Assuming $p(t)$ as the pulse shaping filter and the STE of $\delta_{n} \triangleq \frac{\Delta T_{n}}{T_{s}}$, where $T_{s}$ is a symbol duration and $\Delta T_{n}$ is the amount of deviation from correct sampling time for $n$-th symbol, then considering only the STE effect, the $n$-th received symbol can be expressed as

$$
y_{n}=\sum_{k=1}^{N} x_{k} p\left(n-k+\delta_{n}\right)+w_{n}, \quad n=1,2, \cdots, N,
$$

where, noting that the sampling time offset $\delta_{n}$ is introduced by an oscillator, an accurate model is a Wiener process [1], i.e.,

$$
\delta_{n}=\delta_{n-1}+\eta_{n}, \quad n=2,3, \cdots, N,
$$

with $\eta_{n}$ being i.i.d. zero-mean Gaussian random process with variance of $\sigma_{\eta}^{2}$.

Now, if we consider the effects of PN, CFO and STE at the same time, the $n$-th received symbol can be expressed as follows

$$
y_{n}=\sum_{k=1}^{N} e^{j \Phi_{k}} x_{k} p\left(n-k+\delta_{n}\right)+w_{n}, \quad n=1,2, \cdots, N .
$$

Note that in (3) we have assumed that the received signal is first down-converted at the receiver and then it is sampled by an analog-to-digital converter, which is the procedure that is performed in practice.

Afterwards, we assume that data transmission is done in a pilot-based scenario. It means that if the pilot ratio is $M^{-1}$, in each sub-block of $M$ consecutive symbols, there is one pilot symbol that is known both at the transmitter and at the receiver.

\section{Proposed Extended Kalman Solution}

According to the Wiener process model provided for $\Phi_{n}$ and $\delta_{n}$ in the previous section, we propose the Kalman Filter algorithm for tracking the target error terms based on received symbols and pilot symbols. However, the presented expression for $y_{n}$ in (3) depends on all values of $\Phi_{k}$ for $k=1,2, \cdots, N$, which makes it challenging to track PN and CFO in presence of STE. Consequently, it is more useful to reformulate (3) as follows

$$
y_{n}=e^{j \Phi_{n}} \sum_{k=1}^{N} e^{j \Psi_{k, n}} x_{k} p\left(n-k+\delta_{n}\right)+w_{n},
$$

where $\Psi_{k, n}=\Phi_{k}-\Phi_{n}$. If we express every $\Phi_{i}$ recursively based on $\Phi_{0}, \Delta f$ and $\xi_{i}$ terms, then $\Psi_{k, n}$ will be equal to

$$
\Psi_{k, n}=(k-n) \Delta f+\psi_{k, n},
$$

where $\psi_{k, n}$ is a sum of $|n-k|$ consecutive terms of $\xi_{i}$ and since all $\xi_{i}$ terms are i.i.d Gaussian random variables, $\psi_{k, n}$ is also a zero-mean Gaussian random variable with variance of $\sigma_{k, n}^{2}=|n-k| \sigma_{\xi}^{2}$. Therefore, by substituting $\Psi_{k, n}$ from (5) into (4), the $n$-th received symbol can be expressed as

$$
\begin{aligned}
y_{n} & =e^{j \Phi_{n}} \sum_{k=1}^{N} e^{-j(n-k) \Delta f} e^{j \psi_{k, n}} x_{k} p\left(n-k+\delta_{n}\right)+w_{n} \\
& \simeq e^{j \Phi_{n}} \sum_{k=1}^{N} x_{k} e^{-j(n-k) \Delta f} p\left(n-k+\delta_{n}\right)+\Upsilon_{n}+w_{n},
\end{aligned}
$$

where we have approximated $e^{j \psi_{k, n}}$ by the first two terms of its Taylor expansion and $\Upsilon_{n}$ is defined as follows

$$
\Upsilon_{n}=j e^{j \Phi_{n}} \sum_{k=1}^{N} \psi_{k, n} x_{k} e^{-j(n-k) \Delta f} p\left(n-k+\delta_{n}\right) .
$$

Then, $\Upsilon_{n}$ can be assumed as a zero-mean additive Gaussian noise with variance of

$$
\sigma_{\Upsilon_{n}}^{2}=\bar{P}_{x} \sigma_{\xi}^{2} \sum_{k=1}^{N}|n-k| \mathbf{E}\left\{p^{2}\left(n-k+\delta_{n}\right)\right\},
$$

where the expectation is computed with respect to $\delta_{n}$, which is a zero-mean Gaussian random variable with variance $n \sigma_{\eta}^{2}$. Moreover, we have assumed that transmitted symbols are zero-mean i.i.d. random variables with variance of $\bar{P}_{x}$. In all, the $n$-th received symbol can be expressed as

$$
y_{n}=e^{j \Phi_{n}} \sum_{k=1}^{N} x_{k} e^{-j(n-k) \Delta f} p\left(n-k+\delta_{n}\right)+\dot{w}_{n},
$$

where $w_{n}=\Upsilon_{n}+w_{n}$ is the total additive Gaussian noise with zero-mean and variance of $\sigma_{w_{n}}^{2}=\sigma_{\Upsilon_{n}}^{2}+\sigma_{w}^{2}$.

In the next step, we need to express (9) based on pilot symbols. So, without loss of generality, we imagine that pilot symbols are ordered as $\tilde{x}_{l}=x_{M(l-1)+1}$ for $l=$ 
$1,2, \cdots,\left\lfloor\frac{N}{M}\right\rfloor$, where $\tilde{x}_{k}$ is the $k$-th pilot symbol. Then, the $l$-th received pilot symbol can be expressed as follows

$$
\begin{aligned}
& \tilde{y}_{l}=y_{n}\left.\right|_{n=M(l-1)+1} \\
&=e^{j \tilde{\Phi}_{l}}\left[\sum_{m=1}^{\left\lfloor\frac{N}{M}\right\rfloor} \tilde{x}_{m} e^{-j M(l-m) \Delta f} p\left(M(l-m)+\tilde{\delta}_{l}\right)\right. \\
&\left.+\sum_{\substack{k=1 \\
\forall r: k \neq M(r-1)+1}}^{N} x_{k} e^{-j(n-k) \Delta f} p\left(M(l-1)+1-k+\tilde{\delta}_{l}\right)\right] \\
& \quad+\tilde{w}_{M(l-1)+1},
\end{aligned}
$$

where $\tilde{\Phi}_{l}=\Phi_{M(l-1)+1}$ and $\tilde{\delta}_{l}=\delta_{M(l-1)+1}$ are PN and STE errors for the $l$-th pilot symbol, respectively. In (10), the first summation is over pilot symbols, however, the second summation is over non-pilot (data) symbols which are all unknown at the receiver. Hence, the second summation term in (10) can be considered as an interference term for the $l$-th received pilot.

It is noticeable that our measurement models, expressed in (9) and (10), are not linear functions of $\Phi_{n}, \Delta f_{n}$ and $\delta_{n}$, which means that we need to apply extended Kalman filter (EKF) algorithm in order to be able to track all the target parameters together.

The main difference between measurement models expressed in (9) and (10) is the inter-symbol interference (ISI) term that appears in the measurement equation written for pilot symbols. Therefore, we anticipate that if we use the measurement equation expressed in (9), then we can track PN, CFO and STE errors with much lower mean squared error (MSE). However, in order to be able to use (9) as the measurement model, we need to develop an initial estimation of all transmitted symbols. Hence, our approach in tracking PN, CFO and STE errors based on EKF algorithm is as follows

- Phase I: Apply EKF algorithm based on pilot symbols to track the target parameters and achieve an initial estimation of transmitted symbols.

- Phase II: Apply EKF algorithm based on estimated symbols to achieve both better estimation of the target parameters and improved symbol estimation.

Before discussing the two phases of EKF algorithm, we need to define the state vector of $\mathbf{z}_{n}=\left[\begin{array}{lll}\Phi_{n} & \Delta f_{n} & \delta_{n}\end{array}\right]^{T}$, where we have also considered CFO as a Wiener process, to be able to track it in the Kalman framework. In particular, we adopt the following model

$$
\Delta f_{n}=\Delta f_{n-1}+\nu_{n-1}, \quad n=1,2, \cdots, N .
$$

However, in order to make this model consistent with the assumption made in the previous section where CFO has been modeled as a constant, we consider $\Delta f_{0}$ in (11) equal to the actual CFO value and model $\nu_{n-1} \sim \mathcal{N}\left(0, \sigma_{\nu}^{2}\right)$ using a Gaussian random variable whose variance is much smaller than $\Delta f_{0}$. The value of $\sigma_{\nu}^{2}$ can be seen as a design parameter and should be chosen in a way that keeps $\Delta f_{n}$ close to $\Delta f_{0}$ for all $n=1,2, \cdots, N$. Obviously, appropriate value of $\sigma_{\nu}^{2}$ depends on the size of the block of transmitted symbols, for larger blocks we need to assume smaller values for $\sigma_{\nu}^{2}$. Consequently, based on the defined state vector, system dynamics model can be expressed as follow

$$
\mathbf{z}_{n}=\underbrace{\left[\begin{array}{lll}
1 & 1 & 0 \\
0 & 1 & 0 \\
0 & 0 & 1
\end{array}\right]}_{\triangleq \Lambda}\left[\begin{array}{c}
\Phi_{n-1} \\
\Delta f_{n-1} \\
\delta_{n-1}
\end{array}\right]+\underbrace{\left[\begin{array}{c}
\xi_{n} \\
\nu_{n} \\
\eta_{n}
\end{array}\right]}_{\triangleq \boldsymbol{\epsilon}_{n}}=\boldsymbol{\Lambda} \mathbf{z}_{n-1}+\boldsymbol{\epsilon}_{n} .
$$

In (12), we have assumed that $\xi_{n}, \nu_{n}$ and $\eta_{n}$ are uncorrelated Gaussian random variables for $n=1,2, \cdots, N$, so $\epsilon_{n}$ would be a Gaussian random vector with covariance matrix of $\mathbf{Q}_{n}=$ $\operatorname{diag}\left(\sigma_{\xi}^{2}, \sigma_{\nu}^{2}, \sigma_{\eta}^{2}\right)$.

\section{A. Phase I}

The system dynamics equation represented in (12) is written for all received symbols, but in order to track PN, CFO and STE just based on pilot symbols, we need to also express system dynamics model for pilot symbols. If PN, CFO and STE errors are denoted by $\tilde{\Phi}_{k}, \Delta \tilde{f}_{k}$ and $\tilde{\delta}_{k}$ for $k$-th received pilot symbol, respectively, then,

$$
\begin{aligned}
\tilde{\Phi}_{k}-\tilde{\Phi}_{k-1} & =\Phi_{M(k-1)+1}-\Phi_{M(k-2)+1} \\
& =M \times \Delta \tilde{f}_{k-1} \\
& +\underbrace{\sum_{i=1}^{M-1} i \nu_{M(k-1)+1-i}}_{\triangleq \tilde{v}_{k}}+\underbrace{\sum_{i=0}^{M-1} \xi_{M(k-1)+1-i}}_{\triangleq \tilde{\xi}_{k}}
\end{aligned}
$$

$$
\begin{aligned}
\Delta \tilde{f}_{k}-\Delta \tilde{f}_{k-1} & =\Delta f_{M(k-1)+1}-\Delta f_{M(k-2)+1} \\
& =\underbrace{\sum_{i=0}^{M-1} \nu_{M(k-1)+1-i}}_{=\tilde{\nu}_{k}} \\
\tilde{\delta}_{k}-\tilde{\delta}_{k-1} & =\underbrace{\delta_{M(k-1)+1}-\delta_{M(k-2)+1}}_{=\tilde{\eta}_{k}} \\
& =\underbrace{\sum_{i=0}^{M-1} \eta_{M(k-1)+1-i}}_{i=0}
\end{aligned}
$$

where $\tilde{v}_{k}$ is a linear combination of $(M-1)$ consecutive $\nu_{i}$ terms, $\tilde{\nu}_{k}$ is sum of $M$ consecutive $\nu_{i}$ terms, $\tilde{\xi}_{k}$ is sum of $M$ consecutive $\xi_{i}$ terms, and $\tilde{\eta}_{k}$ is sum of $M$ consecutive $\eta_{i}$ terms. Since $\nu_{i}, \xi_{i}$ and $\eta_{i}$ are white Gaussian noise sequences, $\tilde{v}_{k}, \tilde{\xi}_{k}, \tilde{\nu}_{k}$ and $\tilde{\eta}_{k}$ will also be white Gaussian noise terms with variances of

$$
\begin{array}{lll}
\sigma_{\tilde{v}}^{2}=\frac{M(M-1)(2 M-1)}{6} \sigma_{\nu}^{2} & , & \sigma_{\tilde{\xi}}^{2}=M \sigma_{\xi}^{2} \\
\sigma_{\tilde{\nu}}^{2}=M \sigma_{\nu}^{2} & , & \sigma_{\tilde{\eta}}^{2}=M \sigma_{\eta}^{2},
\end{array}
$$


respectively. Therefore, system dynamics model based on pilot symbols can be expressed as follows

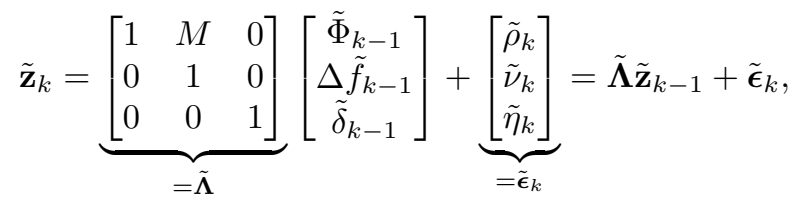

where $\tilde{\mathbf{z}}_{k}=\left[\begin{array}{lll}\tilde{\Phi}_{k} & \Delta \tilde{f}_{k} & \tilde{\delta}_{k}\end{array}\right]^{T}$ is the state vector for $k$-th pilot symbol and $\tilde{\rho}_{k}=\tilde{v}_{k}+\tilde{\xi}_{k}$ is a white Gaussian noise term with variance of,

$$
\sigma_{\tilde{\rho}}^{2}=\frac{M(M-1)(2 M-1)}{6} \sigma_{\nu}^{2}+M \sigma_{\xi}^{2} .
$$

Finally, based on (14) as the system dynamics model and (10) as the measurement model, we should be able to track PN, CFO and STE errors just based on pilot symbols. However, due to the ISI term in (10), it is almost impossible to track the errors without any kind of compensation that allows us to decrease the effect of ISI in our received signal. Fundamentally, if we assume that reliable estimations of PN, CFO and STE are available, then compensated received symbols, indicated by $\hat{y}_{n}$ for $n=1,2, \cdots, N$, can be computed as follows

$$
\hat{y}_{n}=e^{-j \hat{\Phi}_{n}} \sum_{k=1}^{N} y_{k} q\left(n-k-\hat{\delta}_{n}\right),
$$

where $\hat{\Phi}_{n}$ and $\hat{\delta}_{k}$ are the estimated values of PN and STE errors for the $n$-th and the $k$-th received symbols, respectively. Note that $q($.$) is the compensation filter, which can be$ computed according to $p($.$) , such that p(n) * q(n)=\delta(n)$, where $\delta(n)$ is 1 at $n=0$, and is 0 at any non-zero value of $n$.

Consequently, in each step of Kalman framework, we can use our information about the estimated values of PN and STE errors in previous steps to compensate the current erroneous symbol and reduce the effect of ISI term in our measurement model expressed in (10). Considering practical values of pilot ratio, we can assume that PN and STE errors does not change a lot from a pilot symbol to the next pilot. So, the initial estimation of PN and STE errors for each pilot can be taken equal to the estimated parameters for the previous pilot and compensation of the received symbols can be done based on these initial estimations. Therefore, assuming $\operatorname{Sinc}($.$) pulse shaping at the transmitter and the$ receiver, $n$-th compensated received pilot symbol can be expressed as follows

$$
\hat{y}_{n} \simeq e^{-j \hat{\Phi}_{n-1}} \sum_{k=1}^{N} y_{k} \operatorname{Sinc}\left(n-k-\hat{\delta}_{n-1}\right) .
$$

where we have applied $q(n)=\operatorname{Sinc}(n)$ according to the Sinc(.) pulse shaping assumption. By using the compensated symbol of $\hat{y}_{n}$ expressed in (16), as the measurement symbol for each pilot, it would be possible to track $\mathrm{PN}$, CFO

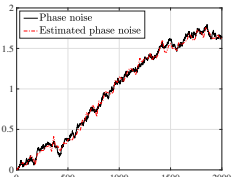

(a) Phase noise

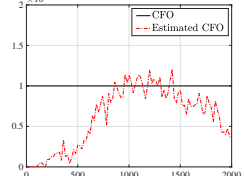

(b) Carrier frequency (c) Sampling time error

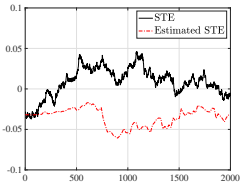
offset
Fig. 2. Estimation of PN, CFO and STE parameters for a block of 2000 symbols, after running Phase $I$ of the proposed algorithm based on pilot symbols.

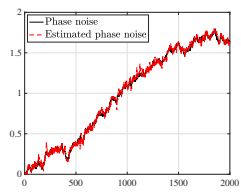

(a) Phase noise
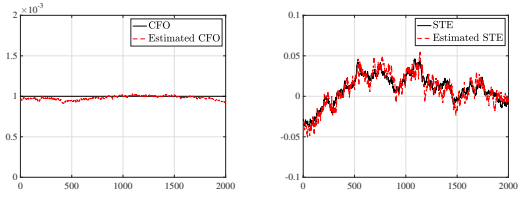

(b) Carrier frequency (c) Sampling time error offset
Fig. 3. Estimation of PN, CFO and STE parameters for a block of 2000 symbols, after running Phase II of the proposed algorithm based on the estimated symbols in Phase I.

and STE errors in the Kalman framework, simultaneously. Then, by using the linear interpolation technique, we can make an initial estimation of PN, CFO and STE errors for non-pilot symbols. According to the available knowledge of initial estimation of target parameters, we can compensate the received symbols according to (15) and then, estimate the transmitted symbols. This initial estimation of transmitted symbols can be used in the next phase of EKF algorithm.

\section{B. Phase II}

After running Phase I of the tracking algorithm, it is expected that we have an initial estimate of transmitted symbols, depicted by $\hat{x}_{n}$ for $n=1,2, \cdots, N$. Then, based on available $\hat{x}_{n} \mathrm{~s}$, it would be possible to apply EKF algorithm over all estimated symbols. It means that in current phase of our algorithm, the EKF runs based on (6) as the measurement equation and (12) as the system dynamics model. However, we need to replace $x_{n}$ symbols in (6) by $\hat{x}_{n} \mathrm{~s}$, namely, the estimated symbols from Phase $I$.

The main corollaries of Phase II of the proposed algorithm are, first, achieving better estimates of PN and STE errors with lower mean squared error (MSE) than the MSE of estimated PN and STE errors in Phase I and second, achieving a more reliable estimation of transmitted symbols in terms of symbol error rate (SER) compared to the achieved SER in Phase I.

\section{NUMERICAL EXAMPLES}

In this section, we conduct several numerical experiments to verify the efficacy of the proposed solution. In the first step, in order to illustrate the performance of provided tracking process, a block of 2000 16-QAM symbols with pilot ratio of $M^{-1}=0.05$ is transmitted through an AWGN channel 


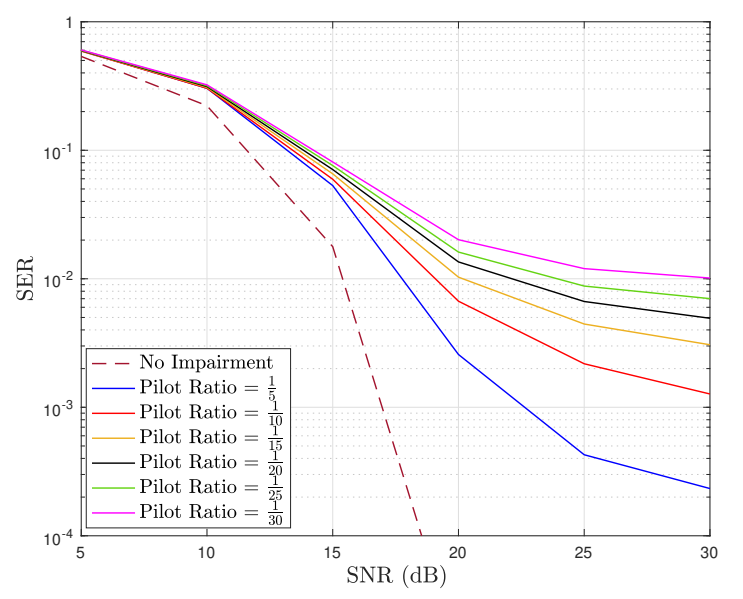

Fig. 4. Symbol error rate versus SNR for different pilot ratios with 16-QAM signals in presence of fixed carrier frequency offset of $10^{-3}$, phase noise with $\sigma_{\xi}^{2}=10^{-4}$ and sampling time error with $\sigma_{\eta}^{2}=10^{-5}$.

with signal-to-noise ratio (SNR), defined as the ratio of data and pilot signal power over noise power, equal to 20 $\mathrm{dB}$ and $\operatorname{Sinc}(t)$ is considered as the pulse shape for data transmission. At the receiver, the received block of symbols is contaminated by PN, CFO and STE, where CFO is assumed to be fixed and equal to $10^{-3}$, PN is modeled by (1) with $\sigma_{\xi}^{2}=10^{-4}$ and STE is modeled by (2) with $\sigma_{\eta}^{2}=2 \times 10^{-6}$. Tracking curves of PN, CFO and STE after running Phase I of the algorithm, are plotted in Fig. 2 for the whole block of symbols. As discussed in section III, it can be observed from Fig. 2 that at least for CFO and STE the pilot-based tracking method is not so reliable, however it seems that we can have a better performance in tracking PN. Nevertheless, an initial estimation of transmitted symbols can be obtained based on the erroneously estimated PN, CFO and STE parameters in Phase I. Thereafter, Phase II of the algorithm is performed based on the estimated transmitted symbols to achieve new estimations of PN, CFO and STE, which are depicted in Fig. 3. Visibly, we can achieve much better trackers for PN, CFO and STE after running Phase II. As a performance metric, we can compare the mean squared error (MSE) of the estimated parameters in Phase I and Phase II of the algorithm. For estimated parameters in Phase I, depicted in Fig. 2, the MSE of PN, CFO and STE are equal to $1.5 \times 10^{-3}$, $1.3 \times 10^{-7}$ and $5.1 \times 10^{-4}$, respectively, while the MSE of estimated parameters in Phase II, depicted in Fig. 3, are equal to $10^{-3}, 5.7 \times 10^{-10}$ and $6.7 \times 10^{-5}$, in the same order. Consequently, if it is desired to achieve more trustworthy estimations of target parameters, performing Phase II of the algorithm would be necessary, however, without Phase I we can not acquire any initial estimation of transmitted symbols to run Phase II based on them.

In the next step, Monte Carlo simulations are performed using 1000 blocks of 10000 16-QAM symbols with the assumption of $\operatorname{Sinc}(t)$ pulse shaping. Fig. 4 depicts the SER

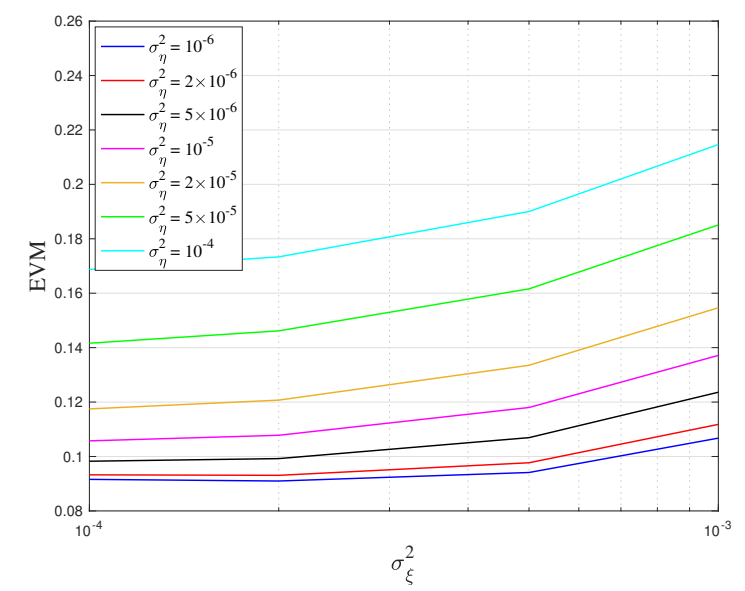

Fig. 5. Error vector magnitude versus $\sigma_{\xi}^{2}$ for different values of $\sigma_{\eta}^{2}$ with CFO of $10^{-3}$, pilot ratio of $M^{-1}=0.05$ and SNR $=20 \mathrm{~dB}$ in a block of 10000 16-QAM symbols.

values versus SNR for different pilot ratios. The variances of PN, STE and frequency offset are set to $\sigma_{\xi}^{2}=10^{-4}$, $\sigma_{\nu}^{2}=10^{-9}$ and $\sigma_{\eta}^{2}=10^{-5}$, respectively. As expected, SER is improved by increasing the pilot ratio, i.e., by sending pilots more frequently. This improved performance is of course obtained at the price of reduced spectral efficiency.

In Fig. 5, we evaluate the error vector magnitude (EVM) of the compensated signal versus variance of the PN, i.e., $\sigma_{\xi}^{2}$, for different values of STE variances, i.e., $\sigma_{\eta}^{2}$. In particular, we compute

$$
\mathrm{EVM}=\sqrt{\frac{\sum_{k=1}^{N}\left|\check{y}_{k}-x_{k}\right|^{2}}{\sum_{k=1}^{N}\left|x_{k}\right|^{2}}},
$$

where $\check{y}_{n}$ is the compensated signal given by

$$
\check{y}_{n}=e^{-j \check{\Phi}_{n}} \sum_{k=1}^{N} y_{k} \operatorname{Sinc}\left(n-k-\check{\delta}_{n}\right),
$$

with final estimated phase and time offset values represented by $\check{\Phi}_{n}$ and $\check{\delta}_{n}$, respectively. The pilot ratio is equal to $M^{-1}=0.05$ and the SNR is set to $20 \mathrm{~dB}$. The figure clearly demonstrates the combined effects of PN and STE on the performance of our proposed solution.

\section{CONCLUSION}

A method for the estimation of sampling time error, phase noise and carrier frequency offset has been proposed using an extended Kalman framework and based on transmitted pilots. Noting that these impairments are highly coupled, the main merit of the proposed solution lies in the fact that it performs the estimation jointly. Proof of concept numerical examples have been provided which demonstrate the feasibility of the proposed tracking algorithm. 


\section{REFERENCES}

[1] J. R. Barry, A. Kavcic, S. W. LcLaughlin, A. Nayak and Wei Zeng, "Iterative timing recovery," in IEEE Signal Process. Mag., vol. 21, no. 1, pp. 89-102, Jan. 2004.

[2] A. A. Nasir, S. Durrani and R. A. Kennedy, "Particle Filters for Joint Timing and Carrier Estimation: Improved Resampling Guidelines and Weighted Bayesian Cramer-Rao Bounds," in IEEE Trans. on Commun., vol. 60 , no. 5, pp. 1407-1419, May 2012.

[3] E. Hosseini and E. Perrins, "Timing, Carrier, and Frame Synchronization of Burst-Mode CPM," in IEEE Trans. on Commun., vol. 61, no. 12, pp. 5125-5138, Dec. 2013.

[4] O. H. Salim, A. A. Nasir, H. Mehrpouyan, W. Xiang, S. Durrani and R. A. Kennedy, "Channel, Phase Noise, and Frequency Offset in OFDM Systems: Joint Estimation, Data Detection, and Hybrid Cramér-Rao Lower Bound," in IEEE Trans. on Commun., vol. 62, no. 9, pp. 33113325, Sept. 2014.

[5] A. A. Nasir, S. Durrani, H. Mehrpouyan, S. D. Blostein, and R. A. Kennedy, "Timing and carrier synchronization in wireless communication systems: A survey and classification of research in the last 5 years," EURASIP J. Wirel. Commun. Netw., vol. 2016, no. 1, p. 180, 2016. 